\title{
A Nontrivial Product in the Stable Homotopy of Spheres
}

\author{
Wang Chong \\ College of Mathematical and Statistics, Cangzhou Normal University, Cangzhou, China
}

Email address:

wangchong_618@163.com

To cite this article:

Wang Chong. A Nontrivial Product in the Stable Homotopy of Spheres. Applied and Computational Mathematics.

Vol. 6, No. 4, 2017, pp. 196-201. doi: 10.11648/j.acm.20170604.17

Received: June 29, 2017; Accepted: July 15, 2017; Published: August 7, 2017

Abstract: Let $p$ be an arbitrary odd prime number greater than eleven and $A$ be the mod $p$ Steenrod algebra. In this paper, it has proved that the product $h_{0} k_{0} \tilde{\delta}_{s+4} \in \operatorname{Ext}_{A}^{s+7,{ }^{*}}\left(Z_{p}, Z_{p}\right)$ is nontrivial and converges to $\alpha_{1} \beta_{2} \delta_{s+4}$ nontrivially of order $p$ in $\pi_{q\left[(s+4) p^{3}+(s+3) p^{2}+(s+4) p+(s+3)\right]-7} S$, where $0 \leq s<p-4, q=2(p-1)$ by making use of the Adams spectral sequence.

Keywords: Steenrod Algebra, Cohomology, May Spectral Sequence, Stable Homotopy of Spheres

\section{Introduction}

Let $A$ be the mod $p$ Steenrod algebra and $S$ be the sphere spectrum localized at an odd prime number $p$. To determine the stable homotogy groups of spheres $\pi_{*} S$ is one of the central problems in homotogy theory. One of the main tools to reach it is the Adams spectral sequence (ASS):

$$
E_{2}^{s, t}=E x t_{A}^{s, t}\left(Z_{p}, Z_{p}\right) \Rightarrow \pi_{t-s} S,
$$

where the $E_{2}^{s, t}-$ term is the cohomology of $A$. So far, not so many family $\zeta_{n-1} \neq 0 \in \pi_{p^{n} q+q-3} S$ for $n \geq 2$ and is represented by $h_{0} b_{n-1} \in \operatorname{Ext}_{A}^{3, p^{n} q+q}\left(Z_{p}, Z_{p}\right)$ has been detected in [1] in the ASS, where $q=2(p-1)$.

To determine the stable homotopy groups of spheres is one of the most important problems in algebraic topology. So far, several methods have been found to determine the stable homotopy groups of spheres. For example, we have the classical Adams spectral sequence (ASS) (cf. [2]) based on the Eilenberg-MacLane spectrum $K Z_{p}$, whose $E_{2}$-term is $\operatorname{Ext}_{A}^{s, t}\left(Z_{p}, Z_{p}\right)$ and the Adams differential is given by

$$
\tilde{d}_{r}: E_{r}^{s, t} \rightarrow E_{r}^{s+r, t+r-1}
$$

where $A$ denotes the mod $p$ Steenrod algebra.
There are three problems in using the ASS: calculation of $E_{2}$ - term $\operatorname{Ext}_{A}^{* * *}\left(Z_{p}, Z_{p}\right)$, computation of the differentials and determination of the nontrivial extensions from $E_{\infty}$ to the stable homotopy groups of spheres. So, for computing the stable homotopy groups of spheres with the classical ASS, we must compute the $E_{2}$-term of the ASS, $\operatorname{Ext}_{A}^{*}{ }^{* *}\left(Z_{p}, Z_{p}\right)$.

Throughout this paper, $p$ denotes an odd prime and $q=2(p-1)$. The known results on $\operatorname{Ext}_{A}^{* * *}\left(Z_{p}, Z_{p}\right)$ are as follows. $\operatorname{Ext}_{A}^{0, *}\left(Z_{p}, Z_{p}\right)$ is trivial by its definition. From [3], $\operatorname{Ext}_{A}^{1, *}\left(Z_{p}, Z_{p}\right)$ has $Z_{p}$ - basis consisting of

$$
a_{0} \in \operatorname{Ext}_{A}^{1,1}\left(Z_{p}, Z_{p}\right), h_{i} \in \operatorname{Ext}_{A}^{1, p^{i} q}\left(Z_{p}, Z_{p}\right)
$$

for all $i \geq 0$ and $\operatorname{Ext}_{A}^{2, *}\left(Z_{p}, Z_{p}\right)$ has $Z_{p}-$ basis consisting of $\alpha_{2}, a_{0}^{2}, a_{0} h_{i}(i>0), g_{i}(i \geq 0), k_{i}(i \geq 0)$,

$b_{i}(i \geq 0)$ and $h_{i} h_{j}(j \geq i+2, i \geq 0)$ whose internal degrees are $2 q+1,2, p^{i} q+1, p^{i+1} q+2 p^{i} q, 2 p^{i+1} q+p^{i} q$ and $p^{i} q+p^{j} q$ respectively.

Let $M$ be the Moore spectrum modulo a prime number $p \geq 3$ given by the cofibration

$$
S \stackrel{p}{\rightarrow} S \stackrel{i}{\rightarrow} M \stackrel{j}{\rightarrow} \sum S
$$


Let $\alpha: \sum{ }^{q} M \rightarrow M$ be the Adams map and $K$ be its cofibre given by the cofibration

$$
\sum{ }^{q} M \stackrel{\alpha}{\rightarrow} M \stackrel{i^{\prime}}{\rightarrow} K \stackrel{j^{\prime}}{\rightarrow} \sum{ }^{q+1} M
$$

where $q=2(p-1)$. This spectrum which we briefly write as $K$ is known to be the Toda-Smith spectrum $V(1)$. Let $V(2)$ be the cofibre of $\beta: \sum{ }^{(p+1) q} K \rightarrow K$ given by the cofibration

$$
\sum{ }^{(p+1) q} K \stackrel{\beta}{\rightarrow} K \stackrel{\bar{i}}{\rightarrow} V(2) \stackrel{\bar{j}}{\rightarrow} \sum{ }^{(p+1) q+1} K
$$

Let $\gamma: \sum q\left(p^{2}+p+1\right) V(2) \rightarrow V(2)$ be the $v_{3}-$ map. As we know, for $t>0$, the $\alpha$ - element $\alpha_{t}=j \alpha^{t} i$, the $\beta-$ element $\beta_{t}=j j^{\prime} \beta^{t} i^{\prime} i$, the $\gamma-$ element $\gamma_{t}=j j^{\bar{j}} \gamma^{t} \bar{i} i^{\prime} i$.

In [4], we have the following results:

(1) For $p \geq 3$ and $t \geq 1, \alpha_{t} \neq 0$ in $\pi_{*} S$.

(2) For $p \geq 5$ and $t \geq 1, \beta_{t} \neq 0$ in $\pi_{*} S$.

(3) For $p \geq 7$ and $t \geq 1, \gamma_{t} \neq 0$ in $\pi_{*} S$.

Studying higher-dimensional cohomology of the mod $p$ Steenrod algebra $A$ is an interesting subject and studied by several authors. For example, In 1980, Aikawa [5] determined $\operatorname{Ext}_{A}^{3, *}\left(Z_{p}, Z_{p}\right)$ by $\lambda-$ algebra. Liu and Zhao [6] prove the following theorem.

Theorem 1.1 For $p \geq 11$ and $4 \leq s<p$, the product $h_{0} b_{0} \tilde{\delta}_{s} \neq 0$ in the classical Adams spectral sequence, where $\tilde{\delta}_{s}$ is given in [7].

\section{The May Spectral Sequence}

The most successful method to compute $\operatorname{Ext}_{A}^{*{ }^{*}}\left(Z_{p}, Z_{p}\right)$ is the MSS. From [8], there is a May spectral sequence (MSS) $\left\{E_{r}^{s, t, *}, d_{r}\right\}$ which converges to $\operatorname{Ext}_{A}^{s, t}\left(Z_{p}, Z_{p}\right)$ with $E_{1}-$ term

$E_{1}^{* *, * *}=E\left(h_{m, i} \mid m>0, i \geq 0\right) \otimes P\left(b_{m, i} \mid m>0, i \geq 0\right) \otimes P\left(a_{n} \mid n \geq 0\right)$

where $E()$ is the exterior algebra, $P()$ is the polynomial algebra,

and $h_{m, i} \in E_{1}^{1,2\left(p^{m}-1\right) p^{i}, 2 m-1}, b_{m, i} \in E_{1}^{2,2\left(p^{m}-1\right) p^{i+1}, p(2 m-1)}, a_{n} \in E_{1}^{1,2 p^{n}-1,2 n+1}$

One has

$$
d_{r}: E_{r}^{s, t, u} \rightarrow E_{r}^{s+1 . t, u-r}
$$

and if $x \in E_{r}^{s, t, *}$ and $y \in E_{r}^{s^{\prime}, t^{\prime},{ }^{*}}$, then

$$
d_{r}(x \cdot y)=d_{r}(x) \cdot y+(-1)^{s} x \cdot d_{r}(y)
$$

In particular, the first May differential $d_{1}$ is given by

$$
\begin{gathered}
d_{1}\left(h_{i, j}\right)=\sum_{0<k<i} h_{i-k, k+j} h_{k, j}, \\
d_{1}\left(a_{i}\right)=\sum_{0 \leq k<i} h_{i-k, k} a_{k}, d_{1}\left(b_{i, j}\right)=0
\end{gathered}
$$

There also exists a graded commutativity in the MSS:

$$
x \cdot y=(-1)^{s s^{\prime}+t t^{\prime}} y \cdot x \text { for } x, y=h_{m, i}, b_{m, i} \text { or } a_{n} .
$$

For each element $x \in E_{1}^{s, t, u}$, we define $\operatorname{dim} x=s$, $\operatorname{deg} x=t, M(x)=u$. Then we have that

$$
\left\{\begin{array}{l}
\operatorname{dim} h_{i, j}=\operatorname{dim} a_{i}=1, \\
\operatorname{dim} b_{i, j}=2, \operatorname{deg} a_{0}=1, \\
\operatorname{deg} h_{i, j}=q\left(p^{i+j-1}+\ldots+p^{j}\right), \\
\operatorname{deg} b_{i, j}=q\left(p^{i+j}+\ldots+p^{j+1}\right), \\
\operatorname{deg} a_{i}=q\left(p^{i-1}+\ldots+1\right)+1, \\
M\left(h_{i, j}\right)=M\left(a_{i-1}\right)=2 i-1, \\
M\left(b_{i, j}\right)=(2 i-1) p,
\end{array}\right.
$$

where $i \geq 1, j \geq 0$.

Note that by the knowledge on the $p-$ adic expression in number theory, for each integer $t \geq 0$, it can be expressed uniquely as $t=q\left(c_{n} p^{n}+c_{n-1} p^{n-1}+\ldots+c_{1} p+c_{0}\right)+e$ where $0 \leq c_{i}<p(0 \leq i<n), p>c_{n}>0,0 \leq e<q$.

For the convenience of writing, we make the following rules:

(i) If $i>j$, we put $a_{i}$ on the left side of $a_{j}$;

(ii) If $j<k$, we put $h_{i, j}$ on the left side of $h_{w, k}$;

(iii) If $i>w$, we put $h_{i, j}$ on the left side of $h_{w, j}$;

(iv) Apply the rules (ii) and (iii) to $b_{i, j}$.

\section{Proof of the Main Theorem}

Before showing the main theorem, we first give some important lemmas which will be used in the proof of it. The first one is a lemma on the representative of $\tilde{\delta}_{s+4}$ in the May spectral sequence.

Lemma 3.1 [7] For $p \geq 11$ and $0 \leq s<p-4$, then the fourth Geerk letter element $\tilde{\delta}_{s+4} \in \operatorname{Ext}_{A}^{s+4, t_{1}(s)}\left(Z_{p}, Z_{p}\right)$ is represented by

$$
a_{4}^{s} h_{4,0} h_{3,1} h_{2,2} h_{1,3} \in E_{1}^{s+4, t_{1}(s), *}
$$

in the $E_{1}-$ term of the May spectral sequence, where $\tilde{\delta}_{s+4}$ is actually $\tilde{\alpha}_{s+4}^{(4)}$ and

$$
t_{1}(s)=q\left[(s+1)+(s+2) p+(s+3) p^{2}+(s+4) p^{3}\right)+s .
$$


By (2), we know that to prove the non-triviality of the product $h_{0} k_{0} \tilde{\delta}_{s+4}$, we have to show that the representative of the product cannot be hit any May differential. For doing it, we give the following two lemma.

Lemma 3.2 Let $p \geq 11$ and $0 \leq s<p-4$. Then we have the May $E_{1}$ - term $E_{1}^{s+6, t(s),{ }^{*}}=0$, where

$$
t(s)=q\left[(s+3)+(s+4) p+(s+3) p^{2}+(s+4) p^{3}\right)+s .
$$

Now we give the proof of the above lemma.

Proof Consider $h=x_{1} x_{2} \ldots x_{m} \in E_{1}^{s+6, t(s),{ }^{*}}$ in the MSS, where $x_{i}$ is one of $a_{k}, h_{r, j}$ or $b_{u, z}, 0 \leq k \leq 4,0 \leq r+j \leq 4$, $0 \leq u+z \leq 3, r>0, j \geq 0, u>0, \quad z \geq 0$. By (5) we can assume that

$$
\operatorname{deg} x_{i}=q\left(c_{i, 3} p^{3}+c_{i, 2} p^{2}+c_{i, 1} p+c_{i, 0}\right)+e_{i}
$$

where $c_{i, j}=0$ or $1, e_{i}=1$ if $x_{i}=a_{k}$, or $e_{i}=0$. It follows

that $\operatorname{dim} h=\sum_{i=1}^{m} \operatorname{dim} x_{i}=s+6$ and

$$
\begin{gathered}
\operatorname{deg} h=\sum_{i=1}^{m} \operatorname{deg} x_{i} \\
=q\left[\left(\sum_{i=1}^{m} c_{i, 3}\right) p^{3}+\left(\sum_{i=1}^{m} c_{i, 2}\right) p^{2}+\left(\sum_{i=1}^{m} c_{i, 1}\right) p+\left(\sum_{i=1}^{m} c_{i, 0}\right)\right]+\left(\sum_{i=1}^{m} e_{i}\right) \\
=q\left[(s+4) p^{3}+(s+3) p^{2}+(s+4) p+(s+3)\right]+s
\end{gathered}
$$

Note that $\operatorname{dim} h_{i, j}=\operatorname{dim} a_{i}=1, \quad \operatorname{dim} b_{i, j}=2$, and $0 \leq s$ $<p-4$. From $\operatorname{dim} h=\sum_{i=1}^{m} \operatorname{dim} x_{i}=s+6$, we can have

$$
m \leq s+6<p+2
$$

Using $0 \leq s+4, s+3, s<p$ and the knowledge on the $p$ -adic expression in number theory, we have that

$$
\left\{\begin{array}{c}
\sum_{i=1}^{m} e_{i}=s \\
\sum_{i=1}^{m} c_{i, 0}=s+3 \\
\sum_{i=1}^{m} c_{i, 1}=s+4 \\
\sum_{i=1}^{m} c_{i, 2}=s+3 \\
\sum_{i=1}^{m} c_{i, 3}=s+4
\end{array}\right.
$$

By $\quad c_{i, 2}=0 \quad$ or $1, \quad$ one has $m \geq s+4$ from $\sum_{i=1}^{m} c_{i, 3}=s+4$.

Note that $m \leq s+6$. Thus $m$ may equal $s+4, s+5, s+6$. Since $\sum_{i=1}^{m} e_{i}=s, \operatorname{deg} h_{i, j} \equiv 0(\bmod q) \quad(i>0, j \geq 0)$, $\operatorname{deg} a_{i} \equiv 1(\bmod q)(i \geq 0) \quad$ and $\quad \operatorname{deg} b_{i, j} \equiv 0(\bmod q)$ $(i>0, j \geq 0)$, then by the graded commutativity of $E_{1}^{* * * *}$ and degree reasons, we can assume that $h=a_{0}^{x} a_{1}^{y} a_{2}^{z} a_{3}^{k} a_{4}^{l} h^{\prime}$ with $h^{\prime}=x_{s+1} x_{s+2} \ldots x_{m}$, where

$$
0 \leq x, y, z, k, l \leq s, x+y+z+k+l=s .
$$

Consequently, we have $h^{\prime}=x_{s+1} x_{s+2} \ldots x_{m} \in E_{1}^{6, t_{2}(s),{ }^{*}}$, where

$$
\begin{aligned}
& t_{2}(s)=q\left[(s+4-l) p^{3}+(s+3-l-k) p^{2}+\right. \\
& (s+4-l-k-z) p+(s+3-l-k-z-y)]
\end{aligned}
$$

Form (6) we have

$$
\left\{\begin{array}{l}
\sum_{i=s+1}^{m} e_{i}=0 \\
\sum_{i=s+1}^{m} c_{i, 0}=s+3-l-k-z-y \\
\sum_{i=s+1}^{m} c_{i, 1}=s+4-l-k-z ; \\
\sum_{i=s+1}^{m} c_{i, 2}=s+3-l-k \\
\sum_{i=s+1}^{m} c_{i, 3}=s+4-l
\end{array}\right.
$$

By the reason of dimension, all the possibilities of $h^{\prime}$ can be listed as $y_{1} \ldots y_{6}, y_{1} \ldots y_{4} z_{1}, y_{1} y_{2} z_{1} z_{2}, z_{1} z_{2} z_{3}$ where the $y_{i}$ is in the form of $h_{r, j}, 0 \leq r+j \leq 4, r>0, j \geq 0$ and $z_{i}$ is in the form of $b_{u, z}, 0 \leq u+z \leq 3, u>0, z \geq 0$.

Case 1. $m=s+4$. From $\sum_{i=s+1}^{s+4} c_{i, 3}=s+4-l$ in (7), we have that $l=s+4-\sum_{i=s+1}^{s+4} c_{i, 3} \geq s$. Thus $l=s, \mathrm{x}=\mathrm{y}=\mathrm{z}=\mathrm{k}=0$.

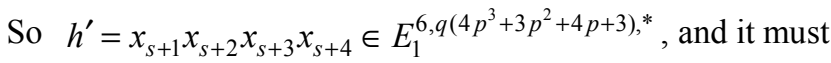
be in the form of $y_{1} y_{2} z_{1} z_{2}$. But because the constant coefficient in $t_{2}(s)$ is $3, h^{\prime}$ is impossible to exist in this case. Then $h$ doesn't exist either. 
Case 2. $m=s+5$. From $\sum_{i=s+1}^{s+5} c_{i, 3}=s+4-l$ in (7), we have that $l=s+4-\sum_{i=s+1}^{s+5} c_{i, 3} \geq s-1$. Thus $l=s-1$ or $s$, and

$$
h^{\prime}=y_{1} \ldots y_{4} z_{1} \in E_{1}^{6, t_{2}(s),{ }^{*}}
$$

(1) If $l=s-1$, then

$$
t_{2}(s)=q\left[5 p^{3}+() p^{2}+() p+(s+3-l-k-z-y)\right]
$$

Because the coefficient of $p^{3}$ is 5 , $h_{4,0}, h_{3,1}, h_{2,2}, h_{1,3}$ and some $b_{u, z}(u+z=3, u>0, z \geq 0$.) must be contained in $h^{\prime}$. So the constant coefficient in $t_{2}(s)$ should be 1 , this is conflict with $s+3-l-k-z-y \geq 3$. $h^{\prime}$ is impossible to exist and then $h$ doesn't exist either. (2) If $l=s$, then $t_{2}(s)=q\left[4 p^{3}+3 p^{2}+4 p+3\right]$.

Because $h^{\prime}=y_{1} \ldots y_{4} z_{1} \in E_{1}^{6, t_{2}(s),{ }^{*}}$, the coefficient of $p^{3}$ is 4 and the constant coefficient in $t_{2}(s)$ is 3 , no matter whether the equation of $u+z=3$ in $z_{1}=b_{u, z}$ is established or not, $h^{\prime}$ is impossible to exist in this case and then $h$ doesn't exist either.

Finally, in order to express this result more intuitively, we list all the possibilities of case 2 in the following table 1 . $h^{\prime}=y_{1} \ldots y_{4} z_{1} \in E_{1}^{6, t_{2}(s),{ }^{*}}$,

Table 1. $m={ }_{S}+5$

\begin{tabular}{llllllll}
\hline possibility & $\mathbf{l}$ & $\mathbf{x}$ & $\mathbf{y}$ & $\mathbf{z}$ & $\mathbf{k}$ & $\boldsymbol{E}_{\mathbf{1}}^{\mathbf{6}, \boldsymbol{t}_{\mathbf{2}}(\boldsymbol{s}),{ }^{*}}$ & $\boldsymbol{h}^{\prime}$ \\
\hline The 1st & $\mathrm{s}-1$ & 1 & 0 & 0 & 0 & $E_{1}^{6, q\left(5 p^{3}+4 p^{2}+5 p+4\right),{ }^{*}=0}$ & Nonexistence \\
The 2nd & $\mathrm{s}-1$ & 0 & 1 & 0 & 0 & $E_{1}^{6, q\left(5 p^{3}+4 p^{2}+5 p+3\right),{ }^{*}=0}$ & Nonexistence \\
The 3rd & $\mathrm{s}-1$ & 0 & 0 & 1 & 0 & $E_{1}^{6, q\left(5 p^{3}+4 p^{2}+4 p+3\right),{ }^{*}=0}$ & Nonexistence \\
The 4th & $\mathrm{s}-1$ & 0 & 0 & 0 & 1 & $E_{1}^{6, q\left(5 p^{3}+3 p^{2}+4 p+3\right),{ }^{*}=0}$ & Nonexistence \\
The 5th & $\mathrm{s}$ & 0 & 0 & 0 & 0 & $E_{1}^{6, q\left(4 p^{3}+3 p^{2}+4 p+3\right),{ }^{*}=0}$ & Nonexistence \\
\hline
\end{tabular}

Case 3. $m=s+6$. From $\sum_{i=s+1}^{s+6} c_{i, 3}=s+4-l$ in (7), we have that $l=s+4-\sum_{i=s+1}^{s+6} c_{i, 3} \geq s-2$. Thus $l=s-2, s-1$ or $s$, and $h^{\prime}=y_{1} \ldots y_{6} \in E_{1}^{6, t_{2}(s),{ }^{*}}$. The coefficient of $p^{3}$ in $t_{2}(s)$ is greater than or equal to 4 . So $h_{4,0}, h_{3,1}, h_{2,2}, h_{1,3}$ must be contained in $h^{\prime}$.

When $l=s-2$ or $s-1$, the coefficient of $p^{3}$ in $t_{2}(s)$ is 6 or 5 and $h^{\prime}=y_{1} \ldots y_{6} \in E_{1}^{6, t_{2}(s),{ }^{*}}$ is impossible to exist. $h$ doesn't exist either.

When $l=s, t_{2}(s)=q\left[4 p^{3}+3 p^{2}+4 p+3\right]$. Because $h^{\prime}=y_{1} \ldots y_{6} \in E_{1}^{6, t_{2}(s),{ }^{*}}, h_{4,0}, h_{3,1}, h_{2,2}, h_{1,3}$ must be contained in $h^{\prime}$ similarly.

But $\operatorname{deg}\left\{h_{4,0} h_{3,1} h_{2,2} h_{1,3}\right\}=q\left[4 p^{3}+3 p^{2}+2 p+1\right]$, in this case $h^{\prime}$ is impossible to exist. Then $h$ doesn't exist either.

We list all the possibilities in the following table.

\begin{tabular}{|c|c|c|c|c|c|c|c|}
\hline possibility & 1 & $\mathbf{x}$ & $\mathbf{y}$ & $\mathbf{z}$ & $\mathbf{k}$ & & $h^{\prime}$ \\
\hline The 1st & $\mathrm{s}-2$ & 2 & 0 & 0 & 0 & $E_{1}^{6, q\left(6 p^{3}+5 p^{2}+6 p+5\right),{ }^{*}}=0$ & Nonexistence \\
\hline The 2nd & $\mathrm{s}-2$ & 0 & 2 & 0 & 0 & $E_{1}^{6, q\left(6 p^{3}+5 p^{2}+6 p+3\right),{ }^{*}}=0$ & Nonexistence \\
\hline The 3rd & $\mathrm{s}-2$ & 0 & 0 & 2 & 0 & $E_{1}^{6, q\left(6 p^{3}+5 p^{2}+4 p+3\right),{ }^{*}}=0$ & Nonexistence \\
\hline The 4th & $\mathrm{s}-2$ & 0 & 0 & 0 & 2 & $E_{1}^{6, q\left(6 p^{3}+3 p^{2}+4 p+3\right),{ }^{*}}=0$ & Nonexistence \\
\hline The 5 th & $\mathrm{s}-2$ & 1 & 1 & 0 & 0 & $E_{1}^{6, q\left(6 p^{3}+5 p^{2}+6 p+4\right),{ }^{*}}=0$ & Nonexistence \\
\hline The 6th & $\mathrm{s}-2$ & 1 & 0 & 1 & 0 & $E_{1}^{6, q\left(6 p^{3}+5 p^{2}+5 p+4\right),{ }^{*}}=0$ & Nonexistence \\
\hline The 7 th & $\mathrm{s}-2$ & 1 & 0 & 0 & 1 & $E_{1}^{6, q\left(6 p^{3}+4 p^{2}+5 p+4\right),{ }^{*}}=0$ & Nonexistence \\
\hline
\end{tabular}

Table 2. $m=s+6$ 


\begin{tabular}{llllllll}
\hline possibility & $\mathbf{l}$ & $\mathbf{x}$ & $\mathbf{y}$ & $\mathbf{z}$ & $\mathbf{k}$ & & $\boldsymbol{h}^{\prime}$ \\
\hline The 8th & $\mathrm{s}-2$ & 0 & 1 & 1 & 0 & $E_{1}^{6, q\left(6 p^{3}+5 p^{2}+5 p+3\right),{ }^{*}=0}$ & Nonexistence \\
The 9th & $\mathrm{s}-2$ & 0 & 1 & 0 & 1 & $E_{1}^{6, q\left(6 p^{3}+4 p^{2}+5 p+3\right),{ }^{*}=0}$ & Nonexistence \\
The 10th & $\mathrm{s}-2$ & 0 & 0 & 1 & 1 & $E_{1}^{6, q\left(6 p^{3}+4 p^{2}+4 p+3\right),{ }^{*}=0}$ & Nonexistence \\
The 11th & $\mathrm{s}-1$ & 1 & 0 & 0 & 0 & $E_{1}^{6, q\left(5 p^{3}+4 p^{2}+5 p+4\right),{ }^{*}=0}$ & Nonexistence \\
The 12th & $\mathrm{s}-1$ & 0 & 1 & 0 & 0 & $E_{1}^{6, q\left(5 p^{3}+4 p^{2}+5 p+3\right),{ }^{*}=0}$ & Nonexistence \\
The 13th & $\mathrm{s}-1$ & 0 & 0 & 1 & 0 & & Nonexistence \\
The 14th & $\mathrm{s}-1$ & 0 & 0 & 0 & 1 & $E_{1}^{6, q\left(5 p^{3}+3 p^{2}+4 p+3\right),{ }^{*}=0}$ & Nonexistence \\
The 15th & $\mathrm{s}$ & 0 & 0 & 0 & 0 & & Nonexistence \\
\hline
\end{tabular}

According to above mentioned analysis, Lamma 3.2 follows.

Lemma 3.3 Let $p \geq 11$ and $0 \leq s<p-4$ then in the cohomology of the $\bmod P$ Steenrod algebra $A$, the product

$$
h_{0} k_{0} \tilde{\delta}_{s+4} \in E x t_{A}^{s+7, t(s)}\left(Z_{p}, Z_{p}\right)
$$

is nontrivial, where

$$
t(s)=q\left[(s+3)+(s+4) p+(s+3) p^{2}+(s+4) p^{3}\right]+s .
$$

Proof Since $h_{1,0}, h_{2,0} h_{1,1}$ and $a_{4}^{s} h_{4,0} h_{3,1} h_{2,2} h_{1,3} \in E_{1}^{*,{ }^{*}, *}$ are permanent cycles in the MSS and converge nontrivially to $h_{0}$, $k_{0}, \tilde{\delta}_{s+4} \in \operatorname{Ext}_{A}^{* * *}\left(Z_{p}, Z_{p}\right)$ for $n \geq 0$, so

$$
h_{1,0} h_{2,0} h_{1,1} a_{4}^{s} h_{4,0} h_{3,1} h_{2,2} h_{1,3} \in E_{1}^{s+7, t(s), *}
$$

is a permanent cycle in the MSS and converges to

$$
h_{0} k_{0} \tilde{\delta}_{s+4} \in E x t_{A}^{s+7,^{*}}\left(Z_{p}, Z_{p}\right) .
$$

From Lamma 3.2, we see that $E_{1}^{s+6, t(s),{ }^{*}}=0$, where

$$
t(s)=q\left[(s+3)+(s+4) p+(s+3) p^{2}+(s+4) p^{3}\right)+s .
$$

Then we can have

$$
E_{r}^{s+6, q\left[(s+3)+(s+4) p+(s+3) p^{2}+(s+4) p^{3}\right],{ }^{*}}=0 \text { for } r \geq 1 .
$$

Thus the permanent cycle

$$
h_{1,0} h_{2,0} h_{1,1} a_{4}^{s} h_{4,0} h_{3,1} h_{2,2} h_{1,3} \in E_{r}^{s+7, t(s), *}
$$

does not bound. That is to say,

$$
h_{1,0} h_{2,0} h_{1,1} a_{4}^{s} h_{4,0} h_{3,1} h_{2,2} h_{1,3} \in E_{r}^{s+7, t(s),{ }^{*}}
$$

can not be hit by any differential in the MSS. It follows that

$$
h_{1,0} h_{2,0} h_{1,1} a_{4}^{s} h_{4,0} h_{3,1} h_{2,2} h_{1,3} \in E_{r}^{s+7, t(s), *}
$$

is a permanent cycle in the May spectral sequence and converge nontrivially to $h_{0} k_{0} \tilde{\delta}_{s+4} \in \operatorname{Ext}_{A}^{s+7,{ }^{*}}\left(Z_{p}, Z_{p}\right)$.
It follows that

$h_{0} k_{0} \tilde{\delta}_{s+4} \neq 0 \in \operatorname{Ext}_{A}^{s+7, q\left[(s+3)+(s+4) p+(s+3) p^{2}+(s+4) p^{3}\right]+s,{ }^{*}}\left(Z_{p}, Z_{p}\right)$ and the theorem is proved.

Lemma 3.4 Let $p \geq 11,0 \leq s<p-4$ and $2 \leq r \leq s+7$, then the groups

$$
\operatorname{Ext}_{A}^{s+7-r, q\left[(s+3)+(s+4) p+(s+3) p^{2}+(s+4) p^{3}\right]+s-r+1}\left(Z_{p}, Z_{p}\right)=0 .
$$

Proof The proof is divided into two parts.

Case 1. $r=s+7$. In this case,

$$
\operatorname{Ext}_{A}^{s+7-r, q\left[(s+3)+(s+4) p+(s+3) p^{2}+(s+4) p^{3}\right]+s-r+1}\left(Z_{p}, Z_{p}\right)=0
$$

by $q\left[(s+3)+(s+4) p+(s+3) p^{2}+(s+4) p^{3}\right]-6>0$ [3]).

Case 2. $2 \leq r \leq s+6$.

To prove that $\operatorname{Ext}_{A}^{s+7-r, t^{\prime \prime}}\left(Z_{p}, Z_{p}\right)=0$, it suffices to prove that $E_{1}^{s+7-r, t^{\prime \prime},{ }^{*}}=0$ in the MSS $[9,10]$, where

$$
t^{\prime \prime}=q\left[(s+3)+(s+4) p+(s+3) p^{2}+(s+4) p^{3}\right]+s-r+1
$$

Consider $h=x_{1} x_{2} \ldots x_{m} \in E_{1}^{S+7-r, t^{\prime \prime},{ }^{*}}$ in the MSS, where $x_{i}$ is one of $a_{k}, h_{r, j}$ or $b_{u, z}, 0 \leq k \leq 4,0 \leq r+j \leq 4$, $0 \leq u+z \leq 3, r>0, j \geq 0, u>0, z \geq 0$. Assume that

$$
\operatorname{deg} x_{i}=q\left(c_{i, 3} p^{3}+c_{i, 2} p^{2}+c_{i, 1} p+c_{i, 0}\right)+e_{i}
$$

where $c_{i, j}=0$ or $1, e_{i}=1$ if $x_{i}=a_{k}$, or $e_{i}=0$. It follows that $\operatorname{dim} h=\sum_{i=1}^{m} \operatorname{dim} x_{i}=s+7-r$ and

$$
\operatorname{deg} h=\sum_{i=1}^{m} \operatorname{deg} x_{i}
$$

$$
\begin{gathered}
=q\left[\left(\sum_{i=1}^{m} c_{i, 3}\right) p^{3}+\left(\sum_{i=1}^{m} c_{i, 2}\right) p^{2}+\left(\sum_{i=1}^{m} c_{i, 1}\right) p+\left(\sum_{i=1}^{m} c_{i, 0}\right)\right]+\left(\sum_{i=1}^{m} e_{i}\right) \\
=q\left[(s+4) p^{3}+(s+3) p^{2}+(s+4) p+(s+3)\right]+s-r+1
\end{gathered}
$$


Note that $\operatorname{dim} x_{i}=1$ or 2 , we get that

$$
m \leq \operatorname{dim} h=s+7-r \leq s+5 \leq p-5+5=p .
$$

By the knowledge about $p$-adic expression in number theory, we have

$$
\begin{gathered}
\sum_{i=1}^{m} c_{i, 3}=s+4, \sum_{i=1}^{m} c_{i, 2}=s+3, \sum_{i=1}^{m} c_{i, 1}=s+4, \\
\sum_{i=1}^{m} c_{i, 0}=s+3, \sum_{i=1}^{m} e_{i}=s-r+1 .
\end{gathered}
$$

When $r>3$, we can get $\operatorname{dim} h=s+7-r<s+4$.

Meanwhile, we have $m \geq s+4$ from $\sum_{i=1}^{m} c_{i, 3}=s+4$, so $\operatorname{dim} h \geq s+4$. This is a contradiction.

When $r=3$, we can get $\operatorname{dim} h=s+7-r=s+4$. By an argument similar to that used in the proof of Lamma 3.1,

we can show that

$$
E_{1}^{s+4, q\left[(s+4) p^{3}+(s+3) p^{2}+(s+4) p+(s+3)\right]+(s-2),{ }^{*}=0 .} .
$$

When $r=2$, then we can get $\operatorname{dim} h=s+5, m \leq s+5$. But from $\sum_{i=1}^{m} c_{i, 3}=s+4$, we get that $m \geq s+4$. There are two possiblities: $m=s+4$ or $m=s+5$. By an argument similar to that used in the proof of lamma 3.2, we can prove that $E_{1}^{s+4, q\left[(s+4) p^{3}+(s+3) p^{2}+(s+4) p+(s+3)\right]+(s-1),{ }^{*}}=0$.

\section{Conclusion}

Theorem Let $p \geq 11$, then in the cohomology of the mod $p$ Steenrod algebra $A$, the product

$$
h_{0} k_{0} \tilde{\delta}_{s+4} \neq 0 \in \operatorname{Ext}_{A}^{s+7, t(s)}\left(Z_{p}, Z_{p}\right)
$$

is a permanent cycle in the ASS and converges to $\alpha_{1} \beta_{2} \delta_{s+4}$ nontrivially of order $p$ in

$$
\pi_{q\left[(s+4) p^{3}+(s+3) p^{2}+(s+4) p+(s+3)\right]-7} S,
$$

where $0 \leq s<p-4, q=2(p-1)$.

Proof First, by Lamma 3.3 we know that

$$
h_{0} k_{0} \tilde{\delta}_{s+4} \neq 0 \in \operatorname{Ext}_{A}^{s+7, q\left[(s+3)+(s+4) p+(s+3) p^{2}+(s+4) p^{3}\right]+s,{ }^{*}}\left(Z_{p}, Z_{p}\right)
$$

Next, it is known that $h_{0}, k_{0} \in \operatorname{Ext}_{A}^{*}{ }^{* *}\left(Z_{p}, Z_{p}\right)$ converge nontrivially to the $\alpha$-element $\alpha_{1}$ and the $\beta$-element $\beta_{2}$ in the ASS respctivly. The $\delta$ - element $\delta_{s+4}$ is represented by $\tilde{\delta}_{s+4} \in E x t_{A}^{s+4, q\left[(s+4) p^{3}+(s+3) p^{2}+(s+2) p+(s+1)\right]+s}\left(Z_{p}, Z_{p}\right) \quad$ in the ASS (cf [11]). Then the map $\alpha_{1} \beta_{2} \delta_{s+4} \in \pi_{*} S$ is represented by $h_{0} k_{0} \tilde{\delta}_{s+4} \neq 0 \in \operatorname{Ext}_{A}^{s+7, t(s)}\left(Z_{p}, Z_{p}\right)$, where

$$
t(s)=q\left[(s+3)+(s+4) p+(s+3) p^{2}+(s+4) p^{3}\right)+s .
$$

\section{Acknowledgements}

The reseach was supported by the Youth Foundation of Hebei Educational Committee (QN 2017505). We thank the referees for their time and comments.

\section{References}

[1] Cohen R. L., Odd primary families in stable homotopy theory, Mem. Amer. Math. S oc., 1981, 242: 1-92.

[2] J. F. Adams, Stable Homotopy and Generalised Homology, Chicago: University of Chicago Press, 1974.

[3] A. Liulevicius, The factorizations of cyclic reduced powers by secondary cohomology operations, Mem. Amer. Math. Soc. 42 1962: 1-112.

[4] Miller H. R., Ravenel D. C. and Wilson W. S., Periodic phenome-na in the Adams-Novikov spectral sequence, Ann. of Math., 1977, 106: 469-516.

[5] T. Aikawa, 3-dimensional cohomology of the mod $p$ Steenrod algebra, Math. Scand. 47 (1980), 91--115.

[6] X. Liu and H. Zhao, On a product in the classical Adams spectral sequence, Proc. Amer. Math. Soc. 137 (2009), no. 7, 2489-2496.

[7] X. Wang and Q. Zheng, The convergence of $\tilde{\alpha}_{s+4}^{(n)} h_{0} h_{k}$, Sci. China Ser. A 41 (1998), no. 6, 622-628.

[8] D. C. Ravenel, Complex Cobordism and Stable Homotopy Groups of Spheres, Orlando: Academic Press, 1986.

[9] X. Liu, Some infinite elements in the Adams spetral sequence for the sphere spectrum, J. Math. Kyoto Univ. 48 (2008), 617-629.

[10] Hao Zhao, Xiangjun Wang, Linan Zhong. The convergence of the product $\tilde{\gamma}_{p-1} h_{0} b_{n-1}$ in the Adams spectral sequence. Forum Mathematicum, 2015, 27 (3): 1613-1637.

[11] Zhong Linan, X. Liu. Non-Triviality of the Product $b_{0} k_{0} \tilde{\delta}_{s+4}$ in the Adams Spectral Sequence. Acta Mathematica Scientia, 2014, 34 (2): 274-282. 